\title{
ANALYSIS OF AN EXPONENTIAL EQUATION WITH ORDINAL VARIABLES ${ }^{1}$
}

\author{
J. L. HICKMAN
}

\begin{abstract}
This paper is concerned with the analysis of the equation $x^{y}=y^{z}$, where $x, y, z$ are variables ranging over ordinals, and where both sides of the equation are transfinite in value. The method used for this analysis consists in regarding $y$ as a parameter and $x$ as an independent variable, and determining necessary and sufficient conditions to be placed upon $x$ so that the resulting equation in $z$ has a solution. Extensive use is made of normal form, as well as results in ordinal arithmetic by both Bachmann and Sherman.
\end{abstract}

0 . We are interested in determining the ordinal solutions of the threevariable equation $x^{y}=y^{z}$ in which each side assumes a transfinite value. Our procedure consists in taking $y=\alpha$ as a parameter, and then finding those ordinals $\beta$ for which $\beta^{\alpha} \geqslant \omega$ and the equation $\beta^{\alpha}=\alpha^{z}$ has an ordinal solution. Since the function $z \mapsto \alpha^{z}$ is normal for any given $\alpha>1$, it is obvious that for any given $\beta$, the equation $\beta^{\alpha}=\alpha^{z}$ has at most one solution. This of course would not be the case if we interchanged the roles of $x$ and $z$.

The paper is divided into four sections. This first section is devoted entirely to the introduction of terminology and the statements of a few known results that will be used extensively throughout the remainder of the paper. In the second section we list our results concerning the equation $x^{y}=y^{z}$, and the last two sections are devoted to the proofs of these results.

Lower case Greek letters always denote ordinals. Whilst we do not preclude these from taking finite values, we shall generally use small Latin letters " $i$ ", " $j ", . . .$, , $s "$, " $t$ " for finite ordinals (numbers), and such a letter will invariably denote a number. The first transfinite ordinal is denoted by " $\omega$ ", and we include 0 among the limit ordinals. For any ordinal $\alpha$, we put $I(\alpha)$ $=\max \{\omega \xi ; \omega \xi \leqslant \alpha\}$, and $F(\alpha)=\alpha-I(\alpha)$.

For any ordinal $\alpha>0$, there is a unique number $n$, a unique decreasing $n+1$-sequence $\left(\alpha_{i}\right)_{i \leqslant n}$ of ordinals, and a unique $n+1$-sequence $\left(p_{i}\right)_{i \leqslant n}$ of positive numbers such that

$$
\alpha=\omega^{\alpha} p_{0}+\omega^{\alpha_{1}} p_{1}+\cdots+\omega^{\alpha_{n}} p_{n}=\sum_{i=0}^{n} \omega^{\alpha_{i}} p_{i} .
$$

This is the "normal form" of $\alpha$, and a proof of the Normal Form Theorem may be found in [4, p. 323]. Because of the continual use made of normal

Received by the editors April 4, 1974 and, in revised form, July 10, 1975.

AMS (MOS) subject classifications (1970). Primary 04A10.

${ }^{1}$ This work was done whilst the author was a Research Fellow at the Australian National University. 
form in this paper, we introduce some notation associated with it.

Let $\alpha>0$ have the normal form indicated above. The number $n+1$ is called the "length" of $\alpha$, and is denoted by " $l(\alpha)$ ". For each $i \leqslant n$, we denote $\alpha_{i}, p_{i}$ by " $e_{i}(\alpha)$ ", " $c_{i}(\alpha)$ ", respectively $-e_{0}(\alpha), c_{0}(\alpha)$ are generally known, respectively, as the degree and coefficient of $\alpha$, and for typographical reasons we shall usually omit the subscript 0 . Finally, since the ordinal $\omega^{\alpha_{n}}$ will also figure prominently in some of our results, we shall denote this by " $R(\alpha)$ ". It is clearly a prime component and the smallest positive remainder of $\alpha$. To avoid wearisome repetition, we adopt the convention that whenever any of these notations are used, it is assumed that $\alpha>0$.

The normal form of $\alpha$ could be said to display the "internal structure" of $\alpha$, relative to the particular ordinal $\omega$. Because of its dependence upon this special ordinal $\omega$, we feel that, if possible, references to the internal structure of any ordinal concerned should be eschewed in the statements of theorems; it seems to us that a theorem stated entirely in terms of "intrinsic" properties of ordinals is better (as a rule) both from the viewpoint of elegance and of clarity than one which is not. Hence we have tried to formulate our results in this manner, but unfortunately we cannot claim complete success in this respect, since occasionally abolition of internal structure references can be achieved only at the expense of clarity.

In [1, p. 53], Bachmann gives the following theorem.

For any ordinal $\delta$, put $\iota_{\delta}=0$ if $\delta$ is limit, $\iota_{\delta}=1$ otherwise. Then for any ordinals $\alpha, \beta, \gamma$ such that $\beta+\alpha=\alpha$, we have $(\alpha+\beta)^{\gamma}=\alpha^{\gamma}+\alpha^{I(\gamma)} \theta(F(\gamma))$, where

(i) $\theta(0)=0$ and $\theta(1)=\beta$;

(ii) $\theta(n)=\alpha^{n-1} \beta+\iota_{\beta} \cdot\left(\sum_{k=2}^{n} \alpha^{n-k} \beta\right)$, for $n \geqslant 2$.

Our main use of this theorem will be the determination of the normal form of $\psi^{\gamma}$ in terms of that of $\psi$. For if we put $\alpha=\omega^{e(\psi)} c(\psi)$ and $\beta=\psi-\alpha$, then we have $\psi=\alpha+\beta$ and $\beta+\alpha=\alpha$, and so Bachmann's result can be applied.

The third result that we shall find necessary in our analysis of $x^{y}=y^{z}$ concerns left-divisors of ordinals, and is due to Sherman [3].

Let $\alpha, \beta$ be positive ordinals, and put $n+1=l(\alpha)$. Then $\beta$ is a left-divisor of $\alpha$ if and only if either

(i) $\beta<R(\alpha)$, or

(ii) $\beta=\omega^{e_{j}(\alpha)} p+\sum_{j<k \leqslant n} \omega^{e_{k}(\alpha)} c_{k}(\alpha)$, for some $j \leqslant n$ and some factor $p$ of $c_{j}(\alpha)$.

The following consequence of Sherman's result is almost trivial, but it will be applied sufficiently often in our work to make its explicit statement worthwhile.

Lemma. Let $\alpha, \beta$ be positive ordinals. Then there is a limit ordinal $\lambda>0$ such that $\beta \lambda=\alpha$ if and only if $\beta<R(\alpha)$.

Proof. Assume that $\beta \geqslant R(\alpha)$, and that $\beta \lambda=\alpha$ for some ordinal $\lambda$. Then by Sherman's result there is some $j \leqslant n$ and some factor $p$ of $c_{j}(\alpha)$ such that

$$
\beta=\omega^{e_{j}(\alpha)} p+\sum_{j<k<n} \omega^{e_{k}(\alpha)} c_{k}(\alpha)
$$


where we are setting $n+1=l(\alpha)$. Define the ordinal $\delta$ by

$$
\delta=\left(\sum_{k<j} \omega^{e_{k}(\alpha)-e_{j}(\alpha)} c_{k}(\alpha)\right)+c_{j}(\alpha) / p .
$$

Routine calculation then gives $\beta \delta=\alpha=\beta \lambda$, and so by left-cancellation of $\beta$ we obtain $\delta=\lambda$. Since it is clear that $\delta$ is successor, this proves our lemma in one direction.

Now assume that $\beta<R(\alpha)$ : then by Sherman's result we must have $\beta \delta=\alpha$ for some $\delta$. Now if $\delta$ were successor, then $\beta$ would be a positive remainder of $\alpha$, yielding the contradiction $\beta \geqslant R(\alpha)$. Thus $\delta$ must be limit. This proves our lemma.

1. In this section, we simply state the four theorems whose proofs comprise the rest of this paper.

THEOREM 1. Let $\alpha>1$ be such that $l(\alpha)=1$.

(1) If $\alpha<\omega$, then for any given $\beta \geqslant \omega$, the equation $\beta^{\alpha}=\alpha^{2}$ has an ordinal solution if and only if $\beta=\rho \alpha^{n}$ for some prime component $\rho$ and some number $n$, with $\rho \geqslant \omega$.

(2) If $\alpha \geqslant \omega$, then for any $\beta>1$, the equation $\beta^{\alpha}=\alpha^{z}$ has no ordinal solution if and only if $\beta+\alpha=\alpha=\varepsilon k$ for some epsilon ordinal $\varepsilon$ and some number $k>1$, or $\beta<\omega<\alpha<\omega^{2}$.

THEOREM 2. Let $\alpha=\omega \sigma$ be a limit ordinal with $l(\alpha)>1$.

(1) For any $\beta$ with $1<\beta<\omega$, the equation $\beta^{\alpha}=\alpha^{2}$ has an ordinal solution if and only if $\alpha<\omega^{R(o)}$.

(2) For any $\beta \geqslant \omega$, the equation $\beta^{\alpha}=\alpha^{z}$ has an ordinal solution if and only if $\alpha<\beta^{R(\alpha)}$.

THEOREM 3. Let $\alpha \geqslant \omega$ be a successor ordinal, let the ordinal $\beta>1$ be given, and let $\rho$ be the smallest nontrivial $($ i.e. $\neq 1)$ right-divisor of $\beta$.

Then the equation $\beta^{\alpha}=\alpha^{z}$ has a limit ordinal solution if and only if $\beta=\omega^{\omega \xi}$ for some $\xi>0$ such that $\rho>\alpha$.

THEOREM 4. Let $\alpha, \beta$ be given ordinals, with $\alpha \geqslant \omega$ and successor, and $\beta>1$. Put $\sigma=I(\alpha), m=F(\alpha)$, and define $m^{*}$ to be 1 or $m$ according as $\beta$ is limit or successor.

Finally, define the ordinals $\rho$ and $\tau$ by

$$
\rho=R(\beta), \quad \tau=R\left[e(\beta)\left(\sigma+m-m^{*}\right)+e(\rho)\right] .
$$

Then the equation $\beta^{\alpha}=\alpha^{z}$ has a transfinite successor ordinal solution if and only if there exists $n>0$ such that we have $\alpha<\omega^{\tau}$ and $\rho \alpha^{n}=\beta^{m^{*}}$.

2. In this section we present the proofs of Theorems 1 and 2 , and defer the proofs of the other two results to our next and final section.

Proof of Theorem 1. (1) We put $\alpha=m$, and assume that $z=\gamma$ is a solution of the equation $\beta^{m}=m^{z}$ : from $\beta \geqslant \omega$, we deduce that $\gamma \geqslant \omega$, and can therefore set $\gamma=\omega \zeta+n$ for some ordinal $\zeta>0$, which gives $\beta^{m}=m^{\gamma}$ $=m^{\omega \zeta} m^{n}=\omega^{5} m^{n}$. But this tells us that $l(\beta)=1$. For suppose not; thus $\beta=\omega^{e(\beta)} c(\beta)+\beta^{\prime}$ for some positive $\beta^{\prime}<\omega^{e(\beta)}$, which yields, via Bachmann, 


$$
\beta^{m}=\omega^{e(\beta) m} c(\beta)+\omega^{e(\beta)(m-1)} c(\beta) \beta^{\prime}+\lambda,
$$

where $\lambda<\omega^{e(\beta)(m-1)}$ is some ordinal whose precise value depends upon that of $\iota_{\beta}$. But since $m>1$, we see from this that $l\left(\beta^{m}\right) \geqslant 2$, a contradiction, as $\beta^{m}=\omega^{\zeta} m^{n}$ and $l\left(\omega^{\zeta} m^{n}\right)=1$. This establishes our claim that $l(\beta)=1$, and so $\beta=\omega^{e(\beta)} c(\beta)$. But now $\omega^{\zeta} m^{m}=\beta^{n}=\omega^{e(\beta) m} c(\beta)$, whence $e(\beta) m=\zeta$ and $c(\beta)=m^{n}$. Letting $\rho$ be the prime component $\omega^{e(\beta)}$, we see that $\rho \geqslant \omega$ and $\beta=\rho m^{n}=\rho \alpha^{n}$, which is the required form.

Now assume that $\beta=\rho \alpha^{n}=\rho m^{n}$, for some prime component $\rho \geqslant \omega$ and some number $n$. Now $\rho=\omega^{e(\rho)}$ and $e(\rho) \geqslant 1$; put $\gamma=\omega e(\rho) m+n$. Then we have

$$
\alpha^{\gamma}=m^{\gamma}=m^{\omega e(\rho) m} m^{n}=\omega^{e(\rho) m} m^{n}=\rho^{m} m^{n}=\beta^{m},
$$

and so the equation $\beta^{\alpha}=\alpha^{z}$ has the solution $z=\gamma$.

(2) We first deal with the case in which $\beta$ is finite, say $\beta=m$. Now if $\alpha=\omega$, then of course $m^{\alpha}=\omega=\alpha^{1}$, and so $z=1$ is a solution. Suppose that $\omega<\alpha<\omega^{2}$, whence $\alpha=\omega k$ for some $k>1$. Then $m^{\alpha}=\omega^{k}$, and since $\alpha^{k-1}=\omega^{k-1} k<\omega^{k}<\omega^{k} k=\alpha^{k}$, it is clear that the equation $\beta^{\alpha}=\alpha^{z}$ has no ordinal solution in this case.

Next we consider the case in which $\omega^{2} \leqslant \alpha<\omega^{\omega}$, i.e. in which $\alpha=\omega^{n} k$ for some $n \geqslant 2$ and some $k \geqslant 1$. Then $m^{\alpha}=\omega^{\omega^{n-1} k}$. If we now set $\gamma=\omega^{n-1} k$, we have

$$
\alpha^{\gamma}=\left(\omega^{n} k\right)^{\omega^{n-1} k}=\omega^{\omega^{n-1} k} .
$$

Thus $z=\gamma$ is a solution of the equation $\beta^{\alpha}=\alpha^{z}$.

Finally in the case of $\beta$ being finite, we assume that $\alpha \geqslant \omega^{\omega}$. Then we have $m^{\alpha}=\omega^{\alpha}$. Thus if $z=\gamma$ is a solution of $\beta^{\alpha}=\alpha^{z}$, we must have $\omega^{\alpha}=\alpha^{\gamma}$ $=\omega^{e(\alpha) \gamma} k$, where $k=1$ or $c(\alpha)$ according as $\gamma$ is limit or successor. Hence in fact we must have $k=1$ and $\alpha=e(\alpha) \gamma$.

Conversely, if there is an ordinal $\gamma$ such that $\alpha=e(\alpha) \gamma$, and if $\alpha$ is such that $c(\alpha)=1$ if $\gamma$ is successor, then we have $\alpha^{\gamma}=\omega^{e(\alpha) \gamma}=\omega^{\alpha}=m^{\alpha}$, and thus $z=\gamma$ is a solution of the equation $\beta^{\alpha}=\alpha^{z}$.

Thus it suffices to show that there is an ordinal $\gamma$ such that $\alpha=e(\alpha) \gamma$ and $c(\alpha)=1$ if $\gamma$ is successor if and only if $\alpha=\varepsilon k$ for no epsilon ordinal $\varepsilon$ and number $k>1$. Now as $l(\alpha)=1$, we have $\alpha=\omega^{e(\alpha)} c(\alpha)$, whence $R(\alpha)$ $=\omega^{e(\alpha)}$. Thus we always have $e(\alpha) \leqslant R(\alpha)$, and so by Sherman's result, there is always an ordinal $\gamma$ such that $\alpha=e(\alpha) \gamma$. Furthermore, by our lemma, this ordinal $\gamma$ is successor if and only if $e(\alpha)=R(\alpha)$. Thus our two conditions reduce to the single condition that if $e(\alpha)=\omega^{e(\alpha)}$ then $c(\alpha)=1$. We now show that $e(\alpha)=\omega^{e(\alpha)}$ if and only if $\alpha=\varepsilon n$ for some epsilon ordinal $\varepsilon$ and some number $n \geqslant 1$. This, however, is trivial, for if $e(\alpha)=\omega^{e(\alpha)}$, then $e(\alpha)$ is an epsilon ordinal and $\alpha=e(\alpha) c(\alpha)$; whilst if $\alpha=\varepsilon n$, then since $\varepsilon=\omega^{\varepsilon}$, we must have $e(\alpha)=\varepsilon$ and thus $e(\alpha)=\omega^{e(\alpha)}$. This proves (2) for the case in which $\beta$ is finite, for in this case we obviously always have $\beta+\alpha=\alpha$.

This leaves us with the case in which $\beta \geqslant \omega$, i.e. $e(\beta) \geqslant 1$. Since $\alpha \geqslant \omega$ and $l(\alpha)=1, \alpha$ must be limit, whence Bachmann's theorem tells us that $\beta^{\alpha}=\omega^{e(\beta) \alpha}$ : furthermore, as before we have $\alpha^{\gamma}=\omega^{e(\alpha) \gamma} k, k$ being 1 or $c(\alpha)$, according as the ordinal $\gamma$ is limit or successor. Thus, assuming that $z=\gamma$ is a 
solution of the equation $\beta^{\alpha}=\alpha^{2}$, we see that $k=1$ and $e(\beta) \alpha=e(\alpha) \gamma$. Conversely, if $\alpha$ is such that $e(\beta) \alpha=e(\alpha) \gamma$ for some $\gamma$ and $c(\alpha)=1$ if $\gamma$ is successor, then $z=\gamma$ is a solution of the equation $\beta^{\alpha}=\alpha^{z}$.

Now as $\alpha=\omega^{e(\alpha)} c(\alpha)$ for some $e(\alpha) \geqslant 1$, it is not difficult to see that $R(e(\beta) \alpha)=\omega^{e(e(\beta))+e(\alpha)}$, whence we obtain $e(\alpha) \leqslant e(e(\beta))+e(\alpha)$ $\leqslant R(e(\beta) \alpha)$, and so by Sherman's result there is always an ordinal $\gamma$ such that $e(\beta) \alpha=e(\alpha) \gamma$. Moreover, by our lemma, this ordinal $\gamma$ is successor if and only if $e(\alpha)=R(e(\beta) \alpha)=\omega^{e(e(\beta))+e(\alpha)}$. Thus, analogously to the previous case, we have reduced the condition of $\beta^{\alpha}=\alpha^{2}$ having a solution to the condition that if $e(\alpha)=\omega^{e(e(\beta))+e(\alpha)}$, then $c(\alpha)=1$.

Assume that $e(\alpha)=\omega^{e(e(\beta))+e(\alpha)}$. Since we have of course $e(\alpha) \leqslant \omega^{e(\alpha)}$ $\leqslant \omega^{e(e(\beta))+e(\alpha)}$, this gives $e(\alpha)=\omega^{e(\alpha)}$, whence as before $\alpha=\varepsilon k$ for some $k \geqslant 1$ and some epsilon ordinal $\varepsilon$. Furthermore, $e(e(\beta))+e(\alpha)=e(\alpha)$, i.e. $\omega^{e(e(\beta))}<\omega^{e(\alpha)}=e(\alpha)$, and so $e(\beta) \omega=\omega^{e(e(\beta))} \omega<e(\alpha)$, which gives $\beta \omega$ $=\omega^{e(\beta)} \omega<e(\alpha)$, and thus $\beta+\alpha=\alpha$. Conversely, if $\beta+\alpha=\alpha=\varepsilon k$, then it is clear that $e(e(\beta))+e(\alpha)=e(\alpha)$, whence $\omega^{e(e(\beta))+e(\alpha)}=\omega^{e(\alpha)}=e(\alpha)$. This completes the proof of (2).

Proof of TheOrem 2. (1) We set $\beta=n$. Now if $z=\gamma$ is a solution of $n^{\alpha}=\alpha^{2}$, then we must have $\gamma$ limit (and of course nonzero). For if $\gamma$ is successor, then a routine calculation using Bachmann's theorem gives $l\left(\alpha^{\gamma}\right)$ $\geqslant l(\alpha)>1$, contrary to the fact that $l\left(n^{\alpha}\right)=l\left(\omega^{\sigma}\right)=1$.

Thus we have $n^{\alpha}=\omega^{\sigma}=\alpha^{\gamma}=\omega^{e(\alpha) \gamma}$, whence $\sigma=e(\alpha) \gamma$. Conversely, if the limit ordinal $\gamma>0$ is such that $\sigma=e(\alpha) \gamma$, then $z=\gamma$ is a solution of $n^{\alpha}=\alpha^{z}$. By our lemma, however, there is a limit ordinal $\gamma$ such that $\sigma=e(\alpha) \gamma$ if and only if $e(\alpha)<R(\sigma)$. Thus the equation $n^{\alpha}=\alpha^{z}$ has a solution if and only if $e(\alpha)<R(\sigma)$.

Assume $e(\alpha)<R(\sigma)$. Then of course $\omega^{e(\alpha)}<\omega^{R(\sigma)}$, and so $\omega^{e(\alpha)+1} \leqslant$ $\omega^{R(\sigma)}$. And since $\alpha<\omega^{e(\alpha)+1}$, we obtain $\alpha<\omega^{R(\sigma)}$. Conversely, if $\alpha<\omega^{R(\sigma)}$, then obviously $\omega^{e(\alpha)} \leqslant \alpha<\omega^{R(\sigma)}$, whence $e(\alpha)<R(\sigma)$. This proves (1).

(2) Take $\beta \geqslant \omega$, i.e. $e(\beta) \geqslant 1$, and assume that $z=\gamma$ is a solution of $\beta^{\alpha}=\alpha^{z}$. Since Bachmann's theorem tells us that $l\left(\beta^{\alpha}\right)=1$, we can show as in (1) that $\gamma$ is limit. Thus $\omega^{e(\beta) \alpha}=\beta^{\alpha}=\alpha^{\gamma}=\omega^{e(\alpha) \gamma}$, and hence the equation $\beta^{\alpha}=\alpha^{z}$ has a solution if and only if $e(\alpha)<R(e(\beta) \alpha)$. Now $\alpha$ is limit and $\alpha \geqslant \omega$; thus $R(\alpha)$ is limit and $R(e(\beta) \alpha)=\omega^{e(e(\beta))} R(\alpha)=e(\beta) R(\alpha)$. Thus our condition for a solution reduces to $\omega^{e(\alpha)}<\omega^{e(\beta) R(\alpha)}$, which, as in (1), is equivalent to $\alpha<\omega^{e(\beta) R(\alpha)}$. However, $R(\alpha)$ is limit; thus $\omega^{e(\beta) R(\alpha)}=\beta^{R(\alpha)}$. This proves (2).

3. Proof of Theorem 3. Assume that $z=\gamma$ is a limit ordinal solution of $\beta^{\alpha}=\alpha^{z}$. Then $\alpha^{\gamma}=\omega^{e(\alpha) \gamma}$ and $\beta^{\alpha}=\beta^{I(\alpha)} \beta^{F(\alpha)}=\omega^{e(\beta) I(\alpha)} \beta^{F(\alpha)}$. This shows, by the familiar argument on length, that we must have $\beta=\omega^{e(\beta)} c(\beta)$, whereupon, by equating coefficients and remembering that $F(\alpha) \neq 0$, we further conclude that $c(\beta)=1$, i.e. $\beta=\omega^{e(\beta)}$.

Thus a limit solution of $\beta^{\alpha}=\alpha^{z}$ exists only if $e(\beta) \alpha=e(\alpha) \gamma$, and as the converse also holds, we deduce in the usual manner that a limit solution exists if and only if $e(\alpha)<R(e(\beta) \alpha)$. But as $F(\alpha) \neq 0$, we have $R(e(\beta) \alpha)$ $=R(e(\beta))$.

Assume that $e(\alpha)<R(e(\beta))$. Thus $e(\beta)=e(\alpha) \gamma$ for some limit ordinal 
$\gamma>0$, and so $e(\beta)>0$ is limit. That is, $\beta=\omega^{\omega 5}$ for some $\zeta>0$, and thus has the required form. Furthermore, as $e(\alpha)<R(e(\beta))$, we have

$$
\alpha<\omega^{e(\alpha)+1} \leqslant \omega^{R(e(\beta))}=\omega^{R(\omega \zeta)}=\rho .
$$

Thus our two conditions are necessary for the existence of a limit solution.

Conversely, suppose that $\beta=\omega^{\omega \zeta}$ for some $\zeta>0$, and that $\alpha<\rho$. Then $\beta=\omega^{e(\beta)}$ and $\rho=\omega^{R(e(\beta))}$, whence from $\alpha<\rho$ we deduce immediately that $e(\alpha)<R(e(\beta))$. Hence a limit solution exists, and so our conditions are sufficient. This proves Theorem 3.

Proof of Theorem 4. (a) Suppose that $z=\gamma$ is a successor solution of $\beta^{\alpha}=\alpha^{z}$, and put $\lambda=I(\gamma), n=F(\gamma)$.

(1) Assume that $\beta$ is successor, whence we have $m^{*}=m$. Now $\beta^{\alpha}$ $=\omega^{e(\beta) \sigma} \beta^{m}$ and $\alpha^{\gamma}=\omega^{e(\alpha) \lambda} \alpha^{n}$ : thus, as $\beta^{m}$ and $\alpha^{n}$ are both successor, we must have $e(\beta) \sigma=e(\alpha) \gamma$ and $\beta^{m}=\alpha^{n}$. Since in this case $\rho=1$, this last equation immediately gives us $\beta^{m^{*}}=\rho \alpha^{n}$.

Furthermore, as $e(\rho)=0$, we have $\tau=R(e(\beta) \sigma)$, and the equation $e(\beta) \sigma$ $=e(\alpha) \gamma$ tells us, via our lemma, that $e(\alpha)<\tau$, and hence (as usual) $\alpha<\omega^{\tau}$.

(2) Assume $\beta$ limit, so that $m^{*}=1$. By expanding both $\beta^{\alpha}=\omega^{e(\beta) \sigma} \beta^{m}$ and $\alpha^{\gamma}=\omega^{e(\alpha) \lambda} \alpha^{n}$ into normal form and equating, we see in particular that

$$
e(\beta) \sigma+e(\beta)(m-1)+e_{s-1}(\beta)=e(\alpha) \lambda+e_{r-1}(\alpha),
$$

where $r=l(\alpha), s=l(\beta)$. Since $\alpha$ is successor and $\beta$ is limit, we have $e_{s-1}(\beta)>0=e_{r-1}(\alpha)$; thus $e(\beta) \sigma<e(\alpha) \lambda$. It follows that if we define the ordinal $\delta$ by $\delta=e(\alpha) \lambda-e(\beta) \sigma$, then $\delta>0, \delta$ is limit, and $\beta^{m}=\omega^{\delta} \alpha^{n}$.

We now expand $\beta^{m}$ and $\omega^{\delta} \alpha^{n}$ into normal form and equate. This gives us, for each $i<s$, the equations

$$
c_{i}(\beta)=c_{i}\left(\alpha^{n}\right), \quad e(\beta)(m-1)+e_{i}(\beta)=\delta+e_{i}\left(\alpha^{n}\right) .
$$

We know, however, that

$$
\delta=e(\alpha) \lambda-e(\beta) \sigma=e(\beta)(m-1)+e_{s-1}(\beta),
$$

and hence we obtain, for each $i<s$,

$$
e_{i}(\beta)=e_{s-1}(\beta)+e_{i}\left(\alpha^{n}\right) \text {. }
$$

Thus we see that $\beta=\omega^{e_{s-1}(\beta)} \alpha^{n}$. But obviously $\rho=\omega^{e_{s-1}(\beta)}$ which, since $m^{*}=1$, establishes the condition $\beta^{m^{*}}=\rho \alpha^{n}$. Returning now to the equation

$$
e(\beta) \sigma+e(\beta)(m-1)+e_{s-1}(\beta)=e(\alpha) \lambda,
$$

we see that this is in fact

$$
e(\alpha) \lambda=e(\beta)\left(\sigma+m-m^{*}\right)+e(\rho),
$$

and the inequality $\alpha<\omega^{\tau}$ is derived from this in the usual manner.

(b) We now assume the existence of a number $n>0$ such that $\beta^{m^{*}}=\rho \alpha^{n}$ and $\alpha<\omega^{\tau}$. From this latter condition we deduce that $e(\alpha)<\tau$, and hence that there is a limit ordinal $\lambda>0$ such that $e(\alpha) \lambda=e(\beta)\left(\sigma+m-m^{*}\right)+$ $e(\rho)$. We put $\gamma=\lambda+n$, and show that $\alpha^{\gamma}=\beta^{\alpha}$.

(1) Assume $\beta$ successor. Then $m^{*}=m$ and $\rho=1$, and so $\beta^{m}=\alpha^{n}$ and $e(\alpha) \lambda=e(\beta) \sigma$. Thus we have

$$
\alpha^{\gamma}=\omega^{e(\alpha) \lambda} \alpha^{n}=\omega^{e(\beta) \sigma} \beta^{m}=\beta^{\alpha} .
$$


(2) Assume $\beta$ limit: then $m^{*}=1$, and since $\rho \geqslant \omega$ and is a prime component, our first condition can be written as $\beta=\omega^{e(\rho)} \alpha^{n}$. But now, if we set $\beta^{\prime}=\beta-\omega^{e(\beta)} c(\beta)$, we have

$$
\begin{aligned}
\alpha^{\gamma} & =\omega^{e(\alpha) \lambda} \alpha^{n}=\omega^{e(\beta) \sigma} \omega^{e(\beta)(m-1)} \omega^{e(\rho)} \alpha^{n}=\omega^{e(\beta) \sigma}\left(\omega^{e(\beta)(m-1)} \beta\right) \\
& =\omega^{e(\beta) \sigma}\left[\left(\omega^{e(\beta)} c(\beta)\right)^{m}+\left(\omega^{e(\beta)} c(\beta)\right)^{m-1} \beta^{\prime}\right] .
\end{aligned}
$$

However, since $\beta$ is limit, it is easily seen that

$$
\left(\omega^{e(\beta)} c(\beta)\right)^{m}+\left(\omega^{e(\beta)} c(\beta)\right)^{m-1} \beta^{\prime}=\left(\omega^{e(\beta)} c(\beta)+\beta^{\prime}\right)^{m}=\beta^{m} .
$$

Thus we have $\alpha^{\gamma}=\omega^{e(\beta) \sigma} \beta^{m}=\beta^{\alpha}$. This concludes the proof of Theorem 4 .

We end this analysis of the equation $x^{y}=y^{z}$ with the simple observation that if $\alpha$ is transfinite and successor and $\beta>1$, then the equation $\beta^{\alpha}=\alpha^{2}$ has no finite solution, since $\beta^{\alpha}$ is limit whilst for any $n, \alpha^{n}$ is successor.

General criteria for one ordinal to be a root of another are given in [2].

\section{REFERENCES}

1. H. Bachmann, Transfinite Zahlen, Ergebnisse der Mathematik und ihrer Grenzgebiete, N.F., Heft 1, Springer-Verlag, Berlin, 1955. MR 17, 134.

2. Ph. W. Carruth, Roots and factors of ordinals, Proc. Amer. Math. Soc. 1 (1950), 470-480. MR 12, 166.

3. S. Sherman, Some new properties of transfinite ordinals, Bull. Amer. Math. Soc. 47 (1941), 111-116. MR 2, 255.

4. W. Sierpiński, Cardinal and ordinal numbers, 2nd rev. ed., Monografie Mat., vol. 34, PWN, Warsaw, 1965. MR 33 \#2549.

Department of Mathematics, Institute of Advanced Studies, Australian National University, Canberra, Australia 